\title{
2011 Materials Research Society Fall Meeting highlights state-of-the-art materials research
}

\author{
www.mrs.org/fall2011
}

T he 2011 Materials Research Society (MRS) Fall Meeting was held in Boston from November 28 to December 2. The Meeting Chairs, Cammy R. Abernathy (University of Florida), Paul V. Braun (University of Illinois at Urbana-Champaign), Masashi Kawasaki (University of Tokyo), and Kathryn J. Wahl (Naval Research Laboratory), compiled a program of 47 technical symposia, broadly divided into the areas of energy and the environment, functional materials, nanomaterials, biomaterials, and materials exploration. Alongside the technical sessions, the Meeting offered award presentations, an equipment exhibition, tutorials, professional development functions, public outreach activities, and government seminars on how to obtain funding for research.

A major thread running through the Meeting was how to use state-of-the-art materials science to develop so-called next-generation devices that can be seamlessly integrated into society, from electronics to medical diagnostics. For example, Kavli Lecturer Mark E. Davis of the California Institute of Technology uses materials science to improve the lives of cancer patients. In order to avoid the side effects of chemotherapy as it is currently applied, Davis said he wants to "move the therapy inside the cancer cell, and then release it" by using organic nanoparticles containing the cancer-killing drug.

Davis has tried to pinpoint precisely the size of the nanoparticle that will most effectively deliver the drug over an extended period and then leave the body. Once a solid tumor reaches a size of about $1 \mathrm{~mm}$ in diameter, the tumor needs new blood vessels to continue growing. These new blood vessels typi- cally grow quickly but inefficiently, leaving them "leaky" with holes. These holes can provide a way to get cancerkilling drugs inside the tumor. Eventually, Davis said, the body needs to rid itself of excess nanoparticles that are no longer delivering drugs to prevent undesirable buildup in the body. So the optimal nanoparticle is one that can pass through the leaks in the blood vessels for tumor treatment and through the pores of the kidneys for elimination. From his decades of study, Davis has determined that a $50-\mathrm{nm}$ nanoparticle is optimal; it is the "right size to do the right thing, in the right place, at the right time," he said.

In another talk about using nanodevices for cancer applications, Michael J. Cima of the Massachusetts Institute of Technology proposed implanting diagnostic devices that can perform biopsies. As surgical procedures performed in operating rooms, biopsies can be invasive, especially if they need to be done multiple times during a patient's illness. The Cima group proposes to implant a tiny device inside the tissue using the same needle used for the biopsy. The de- vice can then do further analysis as and when needed by the physician, thereby eliminating the need for additional invasive biopsies.

In a typical process, a biomarker is transported into the device, which interacts with the sensing media to change the magnetic resonance (MR) contrast. The result can be read out on an MRI scan. This idea was initially tested by using cardiac markers in mice, which can be used to detect the possibility for heart attacks. The MRI signal from the implanted device was found to correlate with the magnitude of the heart attack, a result not observed previously.

The devices have also recently been used to measure the degree of hypoxia (oxygen deficiency) in tumor cells. Hypoxic tumors need higher doses of radiation to completely damage the DNA of the tumor cells, and this necessitates higher doses of radiation therapy to get the same result as non-hypoxic tumors. It has therefore become important for physicians to measure the degree of hypoxia during the treatment phase. The Cima group implanted poly(dimethyl siloxane)-based sensors into mice and successfully measured the oxygen response inside an MRI scanner, thereby demonstrating the viability of implantable diagnostic devices.

In the area of infrastructural applications of materials science, plenary speaker Eric J. Amis illustrated a few polymer-based advances made by the United Technologies Research Center

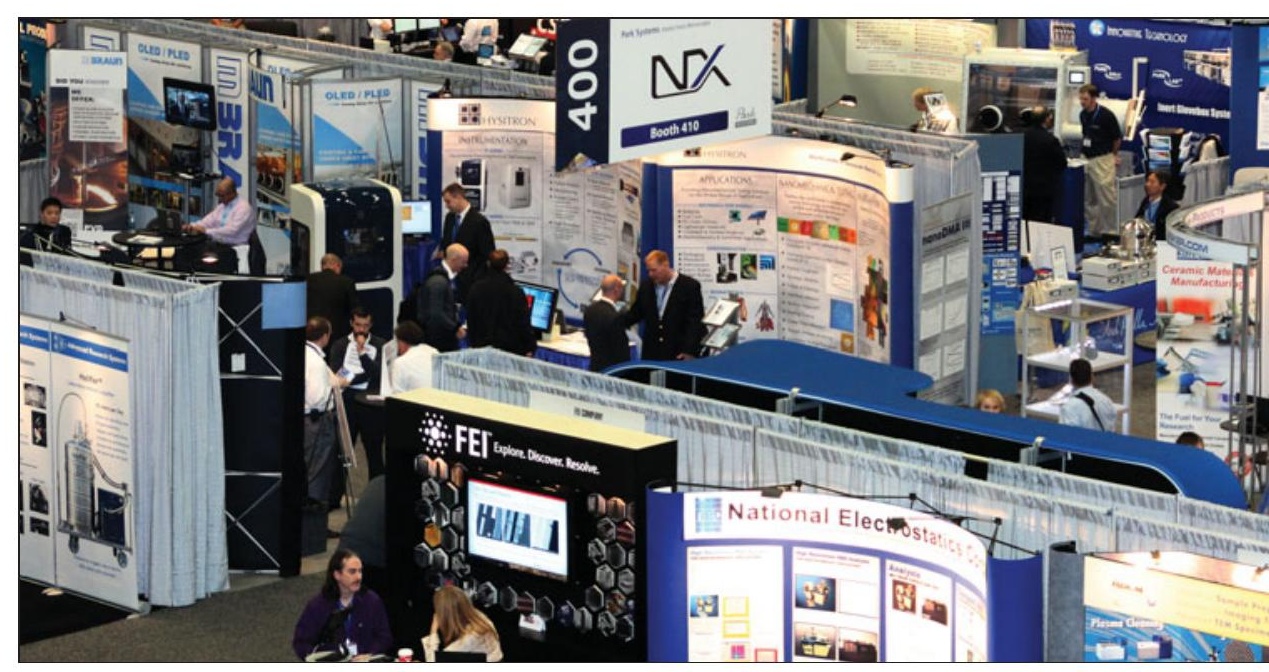



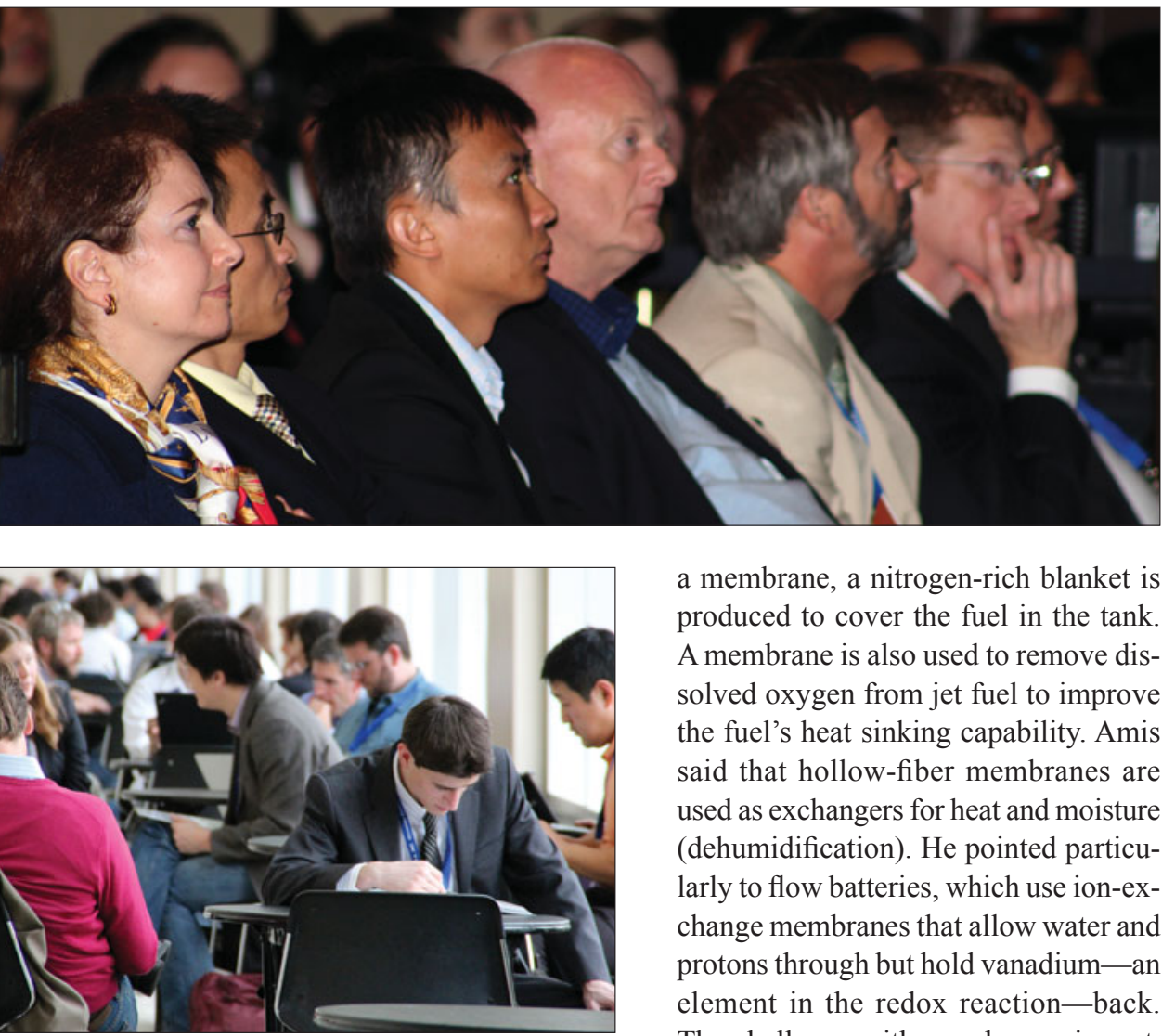

where he is director of the Physical Sciences Department. One of these advances is the flexible polymer belt that the Otis Elevator Company, owned by United Technologies, used to replace steel cables in elevators about 10 years ago. The $30-\mathrm{mm}$ wide by $3-\mathrm{mm}$ thick polyurethane-coated steel belt is more flexible, more durable, and quieter than stainless-steel cables. This innovation made it possible to do away with whole floors at the tops of buildings being devoted to elevator machinery. The challenge in this case was performance: the flexible polymer belt could be made thinner and lighter than the cable used previously. "For some of the tallest buildings in the world, the weight of the rope is the limiting factor," Amis said.

In his discussion of materials as technology enablers, Amis also described two other examples. One example was polymeric membranes, including those being used for aircraft fuel tank inerting to reduce the amount of oxygen above the fuel in a tank. By forcing compressed air into a separation device and allowing the oxygen to permeate out through a membrane, a nitrogen-rich blanket is produced to cover the fuel in the tank. A membrane is also used to remove dissolved oxygen from jet fuel to improve the fuel's heat sinking capability. Amis said that hollow-fiber membranes are used as exchangers for heat and moisture (dehumidification). He pointed particularly to flow batteries, which use ion-exchange membranes that allow water and protons through but hold vanadium - an element in the redox reaction-back. The challenge with membranes is cost, Amis said.

The final topic of the plenary presentation was composites. In bridges, glass fiber-reinforced polymer rebar is being used to replace steel rebar, reducing corrosion and weight while adding strength. Engineers have begun wrapping Kevlar mats around steel rebar for seismic strengthening in earthquake-prone areas. Transport vehicles such as automobiles, boats, and airplanes are using an increasing amount of composites. Much of the new Boeing 787 is built of carbon-reinforced plastic using thermoset materials, Amis said. Metal wind turbine blades, which had diameters of 15 meters in 1985 , have been replaced with composite blades now spanning 126 meters in diameter, with 160 meters expected soon. Composites are used in this application for their light weight, strength, and stiffness.

In the area of electronics, Rodrigo F. Martins of FCTUNI, Portugal, talked about his group's progress in developing solid-state paper batteries. Cellulose is cheap, flexible, lightweight, recyclable, and has good dielectric properties. In both its natural and artificial states, cellulose has properties that make it attractive for use in self-sustained paper-based disposable electronics. Martins' group has already demonstrated a host of applications like paper-based transistors, memory, and electrochromic devices. Developing a paper-based battery to power these devices was a natural extension of their work.

The solid-state paper battery comprises a paper electrolyte sandwiched between thin-film electrodes. Parameters like ion content, porosity, fiber size, hydrophobicity, and hydrophilicity of the paper can be controlled to attain the desired electrochemical behavior. Adsorbed water and hydroxyl ions also help battery operation.

The initial paper battery prototypes produced $3.4 \mathrm{~V}$ and could survive up to $150^{\circ} \mathrm{C}$ without degradation in performance. It was also seen that humidity levels play a key role: short-circuit currents scale directly with increase in humidity. Electrochemical tests reveal that these paper batteries discharge abruptly, but can be recharged to recover up to $80 \%$ of their initial voltage on exposure to $70 \%$ humidity.

Martins said that, in the future, these paper batteries could be used in the cosmetics industry and in medical drug delivery to inject drugs directly into the skin. A plethora of applications is envisaged in other areas like radio frequency (RF) tags, intelligent packaging, disposable toys, and origami electronics, Martins said.

Following are more highlights from the technical sessions as well as coverage of awards and special events.

\section{Energy and the environment}

Organic photovoltaics (OPV) is an emerging PV technology that shows potential for low-cost, lightweight, and flexible solar electricity generation. In the past few years, significant progress has been made with energy conversion efficiencies now in the $8 \%$ range in both polymeric and small moleculebased OPV devices. In Symposium $\mathrm{H}$ on this topic, Sun, a member of Alan J. Heeger's group at the University of 
California-Santa Barbara (UCSB), provided an overview of the state of the art in OPVs. Sun said that starting from $1 \%$ in 1995, the solar energy conversion efficiencies of bulk heterojunction organic (polymer-fullerene-blend-based) solar cells have steadily increased, reaching $9.3 \%$ in 2011 . As the race toward $10 \%$ efficiency continues, many groups are now replacing the light-absorbing donor polymers typically used in these solar cells with small molecules. Problems associated with the polymer-such as batch-to-batch variations in solubility, molecular weight, polydispersity and purity, and processing difficulties-do not apply to these solution-processed small molecules.

The Heeger group recently demonstrated a $7 \%$ efficient small-moleculebased organic solar cell incorporating a PC70BM (a 70-carbon fullerene derivative) acceptor and a donor-acceptor small molecule DTS $\left(\mathrm{PTTh}_{2}\right)_{2}$ as a polymer replacement. Typically, fill factors, defined as the ratio of the actual maximum obtainable power to the theoretical power of a solar cell, are the limiting issue in small-molecule-based organic solar cells, but here they obtain excellent fill factor values exceeding $60 \%$. The efficiencies were also enhanced by adding the right amount of an additive, and by carefully removing all impurities after the small-molecule synthesis.

Sun said that special attention must be given to the role of impurities in these types of solar cells. Less than $1 \%$ impurity content can cause efficiency values to drop from the remarkable $7 \%$ to an average $3 \%$. The reason for this degradation is that impurities lead to trapassisted recombinations which lower photovoltaic parameters.

Throughout the symposium, encouraging progress was reported. Hongbin Wu of South China University of Technology reported an $8.4 \%$ efficiency polymer solar cell. Shinji Aramaki of Mitsubishi Chemical Corporation reported nanostructured solution-processed smallmolecule OPV work related to tetrabenzoporphyrin (BP) and silylmethyl-fullerene, and showed their latest efficiency of $10.0 \%$ certified by AIST on a $1 \mathrm{~cm}^{2}$ sam- ple - the first certified double-digit OPV efficiency. Yang Yang of the University of California-Los Angeles showcased a solution-processed tandem structure that successfully minimizes photovoltage loss and enables higher device efficiency. An NREL-certified 8.6\% tandem efficiency on a laboratory-scale device was shown, and Yang said that higher performance has been achieved. Several academic groups (including the University of Florida and the University of Akron) and companies (including Konarka, Solarmer, and Sumitomo) also showed over $8 \%$ OPV results with Karsten Walzer of Heliatek hinting at above 9\% for their latest vacuum-processed tandem device. Gui Bazan from UCSB showed over 7\% efficiency for a solution-processed small-molecule OPV.
The performance progress comes from not only materials, but also interface engineering, such as solution-processed metal oxides, polyelectrolyte, and inverted and tandem structure. Jinsong Huang (University of Nebraska-Lincoln) expanded their OPV work using ferroelectric polymer as an interface layer to achieve both improved efficiency and introduce new functionality.

Harry Atwater of the California Institute of Technology addressed a topic, in Symposium J, at the forefront of current solar energy research where he surprisingly began with the thermodynamics of photonics. What he called this "rather old work" laid the foundation of limiting theories (like the 1961 ShockleyQueisser efficiency limit), which to this day haunt those working on increasing

\section{Materials Research Society Fall Meeting Symposium Support}

1-Material, Inc

3M Company

Advanced Diamond Technologies, Inc.

Agilent Technologies, Inc.

Air Force Research Laboratory

Anasys Instruments

Angstrom Engineering, Inc.

Applied Diamond, Inc.

Applied Materials, Inc

Army Research Office

Asylum Research

Australian Nuclear Science

and Technology Organization

Bayer USA Foundation

Beneq Oy

Cameca Instruments

CEA-LIST

Covidien

CrysTec GmbH

Defense Threat Reduction Agency

Department of Energy,

Office of Basic Energy Sciences

Elsevier

FEl Company

General Electric Global Research

Hasselt University

Helmholtz-Zentrum Geesthact

Institute of Polymer Research

Hitachi High Technologies America

HORIBA Jobin Yvon, Inc.,

A HORIBA Scientific Company

Hysitron, Inc.

IBM Research

IBM Research-Zurich

JEOL Ltd.

Journal of Materials Chemistry,

Royal Society of Chemistry

Karlsruhe Institute of Technology

The Kurt J. Lesker Co.
Lawrence Livermore National Laboratory Energetic Materials Center

LifeCell Corp.

Mantis Deposition Ltd.

MEMS Industry Group, Inc.

Micron Technology Foundation, Inc.

Micro Photonics, Inc.

The Multi-Scale Technologies Institute

Nanomaterials \& Nanofabrication Labs

Nanomechanics, Inc.

Nanonics Imaging Ltd.

NanoRods, LLC

NanoWorld AG

National Institute

for Materials Science (NIMS)

National Science Foundation

National Science Foundation, CMMI/

Nanomanufacturing

NIL Technology

NOVOLED AG

Oak Ridge National Laboratory

Office of Naval Research

Omicron NanoTechnology GmbH

Picosun USA, LLC

Radiant Technologies, Inc

Sandia National Laboratories

Schlumberger

Seki Technotron

Silex

sp3, Inc.

SPECS Surface Nano Analysis GmbH

STAIB Instruments, Inc.

TE Connectivity

Teleflex Medical OEM

Texas Instruments

Veeco Instruments

World Gold Council

Zurich Instruments Ltd. 


\section{Poster prizes awarded at 2011 MRS Fall Meeting}

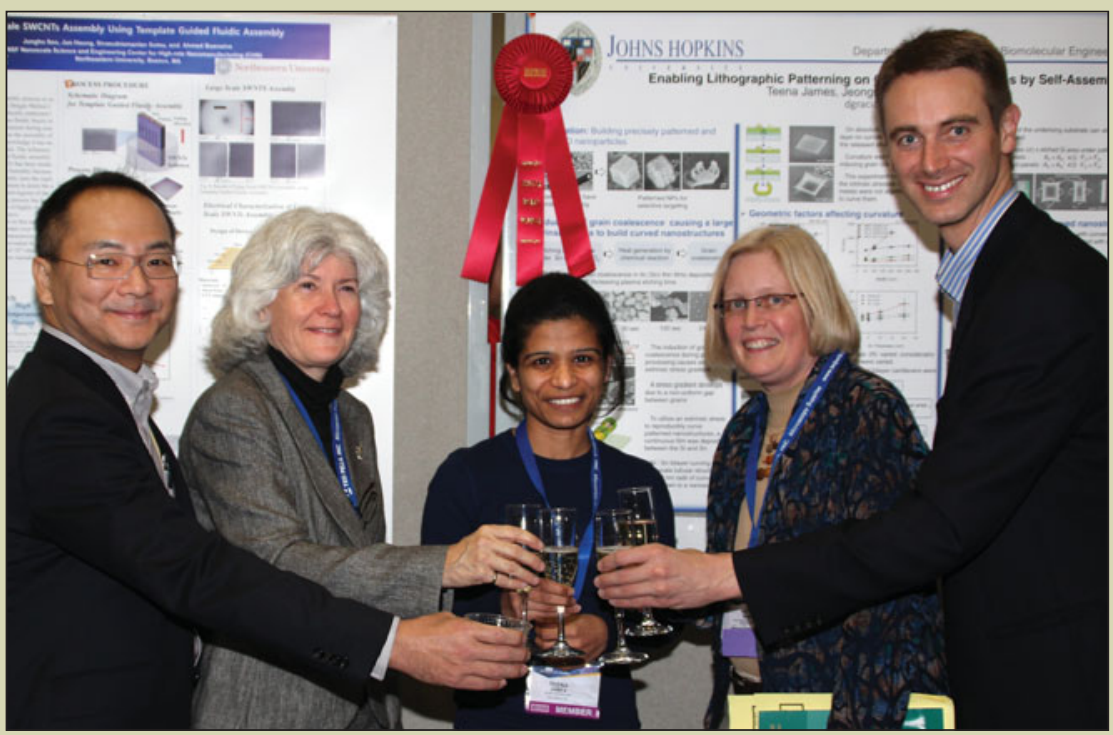

The 2011 Fall Meeting Chairs awarded prizes for the following best poster presentations: (E3.4) Photoelectrochemical Water Splitting Using Copper Gallium Chalcogenides, Tsutomu Minegishi, Jaehong Kim, Makoto Moriya, Jun Kubota, and Kazunari Domen of the University of Tokyo, Japan; (F4.18) Interweaving Lithium Ion Battery Cathodes with Graphene by Genetic Engineering of M13 Virus, Dahyun Oh, Xiangnan Dang, Hyunjung Yi, Mark A. Allen, and Angela M. Belcher of the Massachusetts Institute of Technology, USA and the David H. Koch Institute for Integrative Cancer Research, USA; Kang Xu of the U.S. Army Research Laboratory, USA; and Yun Jung Lee of Hanyang University, South Korea; (J7.39) Compositional Variations in Nanoimprinted Bulk Heterojunction Organic Photoactive Layers Used in Light Trapping Device Architectures, John Tumbleston, Brian Collins, and Harald Ade of North Carolina State University, USA; Abay Gadisa, Edward Samulski, and Rene Lopez of the University of North Carolina, Chapel Hill, USA; (O7.42) Admittance Spectroscopy of Hole States in GaSb/GaAs Quantum Dots with Type-II Band Alignment, Jinyoung Hwang, Andrew J. Martin, Joanna Millunchick,

and Jamie D. Phillips of the University of Michigan, Ann Arbor, USA; (P9.19) The Role of Intermediate Polarization Variants on Domain Switching Behavior in Epitaxial $\mathrm{BiFeO}_{3}$ Thin Films Investigated by Angle-Resolved Piezoresponse Force Microscopy, Moonkyu Park and Kwangsoo No of KAIST, South Korea; and Seungbum Hong, Jeffrey A. Klug, and Orlando Auciello of Argonne National Laboratory, USA; (P13.39) Cross Sectional Ferroelectric Domain Evolution in Ferroelectric Thin Films by In Situ TEM, Christopher T. Nelson, Peng Gao, Jacob R. Jokisaari, and Xiaoqing Pan of the University of Michigan, Ann Arbor, USA: Colin Heikes, Carolina Adamo, Alexander Melville, and Darrell G. Schlom of Cornell University, USA; Benjamin Winchester, Yijia Gu, and Long-Qing Chen of The Pennsylvania State University, USA; Yuanming Liu and Jiangyu Li of the University of Washington, Seattle, USA; and Seung-Hyub Baek, Chad M. Folkman, and Chang-Beom Eom of the University of Wisconsin, Madison, USA; (T5.25) $\mathrm{SnO}_{2}$ Nanowire Inverter Arrays on Hemispherical Deformable Substrates, Gunchul Shin, Min Young Bae, Sahng Ki Hong, Junghwan Huh, Chang Hoon Yoon, Gyu-Tae Kim, and Jeong Sook Ha of Korea University, South Korea; and John A. Rogers of the University of Illinois at Urbana-Champaign, USA; (U8.24) Oligoaniline Crystals: Morphology Control, Hierarchical Assembly and Structure-Property Relationships, Yue Wang and Richard B. Kaner of the University of California, Los Angeles, USA and the California NanoSystems Institute, USA; Henry D. Tran of Fibron Technologies, Inc., USA; and Jinglin Liu and David C. Martin of the University of Delaware, USA; (U13.36) High-Performance $\boldsymbol{n}$-Type Organic Field-Effect Transistors Based on Push-Pull Styryl $\pi$-Conjugated Frameworks, Jong H. Kim, Sun Woo Yun, Seunghoon Shin, and Soo Young Park of Seoul National University, South Korea; Hoichang Yang of Inha University, South Korea; Byeong-Kwan An of The Catholic University of Korea, South Korea; and Young Bin Lee and Jong-Hyun Ahn of Sungkyunkwan University, South Korea; (V12.22) Multifunctional Microvascular Composites Fabricated from Coated Sacrificial Fibers (CSFs), Hefei Dong, Sarut Chayanupatkul, Nancy Sottos, Scott White, and Jeffrey Moore of the University of Illinois at Urbana-Champaign, USA; and Aaron Esser-Kahn of the University of California, Irvine, USA; (FF7.5) Enabling Lithographic Patterning on Curved Nanostructures by Self-Assembly, Teena James, Jeong-Hyun Cho, and David H. Gracias of Johns Hopkins University, USA; (MM10.8) Effect of Atomic-Scale Topography and Ligand Density on Nanoparticle-Adsorbed Protein Structure and Function, Jennifer E. Gagner, Xi Qian, Jonathan S. Dordick, and Richard W. Siegel of Rensselaer Polytechnic Institute, USA; (NN3.19) The Assembly of Gold Nanoparticles on DNA: Effect of Ligands Charge and Polarity, Yaroslava G. Yingling and Abhishek Singh of North Carolina State University, USA; (O06.6) Ultrastructural Origins of Optical Properties in the Exoskeletons of Beetles, Xia Wu, Andreas Erbe, Helge O. Fabritius, and Dierk Raabe of Max-Planck-Institut für Eisenforschung GmbH, Düsseldorf, Germany; (RR4.6) Chemical Changes Underlying Aging of Silica in Nanoscale Frictional Contacts, Yun Liu and Izabela Szlufarska of the University of Wisconsin, Madison, USA. the power-conversion efficiency of solar cells. These efficiency limits can be traced to a variety of sources of entropy gain that occur during the light absorption and energy-conversion process.

The main message from Atwater's talk was ways to design an optimum solar cell that minimizes these losses. Three factors are of importance, namely, high internal quantum efficiency, control of radiative emission angles, and density of states. Novel light collimation techniques like close-packed arrays of microphotonic collimators can significantly reduce emission angles, thereby reducing phonon entropy. The traditional $4 n^{2}$ light trapping limit, which gives the maximum concentration of light within the cell, where $n$ is the refractive index of the solar cell, will no longer suffice as solar cells get thinner and efficiency values need to climb higher. The Atwater group showed that by generating structures that manipulate the density of states in photonic materials, they could achieve light trapping levels that exceed traditional ray-optics-based approaches. The whispering gallery dielectric nanospheres for light trapping are an example that showed significant absorption enhancements in thin solar cells.

In reference to a recent demonstration of a record $28 \%$ efficient, single-junction GaAs solar cell by Alta Devices, Atwater said, "I see no reason why working together we cannot attain 50-70\% efficiencies in the next 10 years or so."

While solar conversion efficiencies of these magnitudes are attention grabbing, efficiency is not the only important property that materials scientists interested in electronics are investigating. In a Symposium F presentation on mobile energy, Manish Chhowalla of Rutgers University made the case for enhancing the flexibility of energy devices, announcing that "reduced graphene oxide (rGO) is a flexible material for flexible electronics." Chhowalla's group has already demonstrated their ability to tune graphene oxide chemistry by chemical synthesis routes. In this regard, removing oxygen groups is critical, particularly to improve electrical conductivity. By 
pioneering new routes for $\mathrm{O}_{2}$ removal, a variety of applications were made possible. Among them were light-emitting electrochemical cells (LEC) that use GO as solution-processed electrodes on flexible substrates.

These GO layers were also demonstrated as hole-transport layers (HTLs) to replace the corrosive PEDOT:PSS-based HTLs. Chhowalla's group also replaced the platinum-based counter electrode used in dye-sensitized solar cells (DSSC) by an electrode comprised of rGO-metal hybrid nanoparticles. Furthermore, solution-processed GO was used to demonstrate $\mathrm{CO}_{2}$ reduction and low-voltage thin-film transistors (TFTs) using selfassembled monolayers (SAMs).

Not to be outdone by solar cells, fuel cells were the topic of discussion in Symposium B. James E. McGrath of Virginia Polytechnic Institute and State University elucidated the desired properties of a practical proton-exchange membrane (PEM): high proton conductivity at ambient humidity, good mechanical properties in wet and dry conditions, low fuel and oxidant permeability, and oxidative and hydrolytic stability. The major technological challenge is to increase the operating temperature from the current value of $80^{\circ} \mathrm{C}$ to the $100-120^{\circ} \mathrm{C}$ range, while decreasing the relative humidity $(\mathrm{RH})$ operating range from the current value of $80-100 \%$. Toward this end, he and his colleagues at Virginia Tech are investigating sulfonated dichloro diphenylsulfone monomers (SDCDPS) as elements in block copolymers for use as PEMs.

By carefully creating a nanophaseseparated morphology with a sharp interface between the hydrophilic and hydrophobic blocks of the copolymer, they were able to increase the water selfdiffusion coefficient and obtain better performance of the PEM at lower RH. In one experiment, the block copolymer performed comparably to the standard Nafion PEM at $100^{\circ} \mathrm{C}$ and $40 \% \mathrm{RH}$; it far exceeded the performance of a random polymer PEM. The researchers found three types of water in these systems, depending on the morphology of the copolymer: (1) non-freezable, tightly bound water; (2) freezable, loosely bound wa- ter, with a broad melting behavior; and (3) free water, with the normal sharp freezing point at $0^{\circ} \mathrm{C}$. By annealing the material above the $\mathrm{T}_{\mathrm{g}}$ of the hydrophobic phase, the copolymer morphology self-assembled more quickly, and the material showed more ductility as $\mathrm{RH}$ increased.

\section{Functional materials}

Oxides are key constituents in modern electronics. Presenters in Symposium M were interested in taking oxide semiconductor materials from the laboratory to commercialization, where they can play active roles in electronic devices such as light-emitting diodes (LEDs), laser diodes, high-speed transistors, low-cost transistors for flat-panel displays, sensors, and photovoltaics/solar cells.

Semiconducting oxides show tremendous potential for electronics and photonics, areas in which research activity and device development are experiencing rapid growth. Numerous presentations were given on crystalline and amorphous oxide thin-film materials, including $\mathrm{ZnO}$, $\mathrm{InGaZnO}, \mathrm{SnO}_{2}$, and

\section{Graduate Students Receive Gold and Silver Awards}

Graduate Student Awards were announced during an evening ceremony on November 30, at the 2011 Materials Research Socety Fall Meeting in Boston.

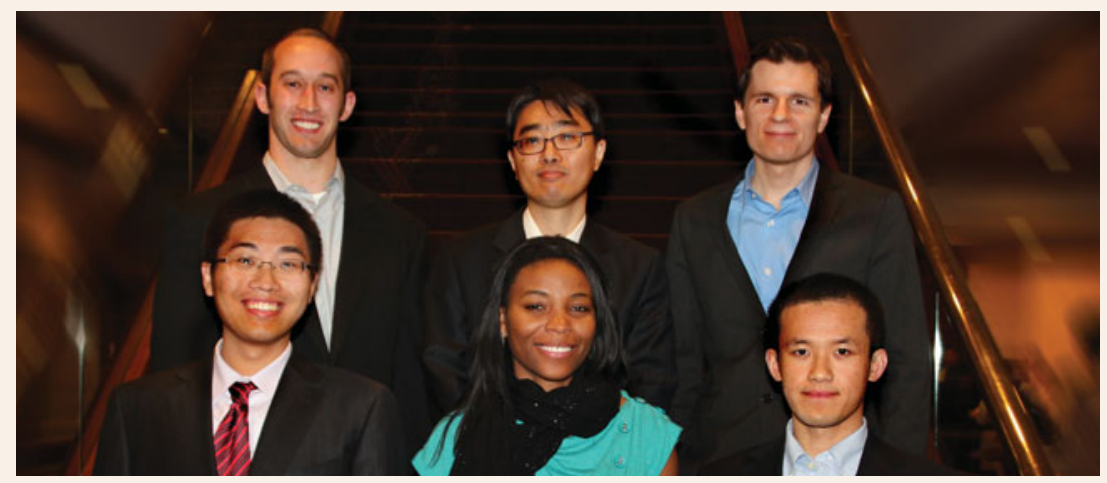

Gold Graduate Student Awards were awarded to (front row, left to right): Shu Hu (Stanford University), Luisa Whittaker (State University of New York at Buffalo), and Jiamian Hu (Tsinghua University); (back row, left to right): Matthew Jones (Northwestern University), Sung Hoon Kang (Harvard University), and Robert Macfarlane (Northwestern University).

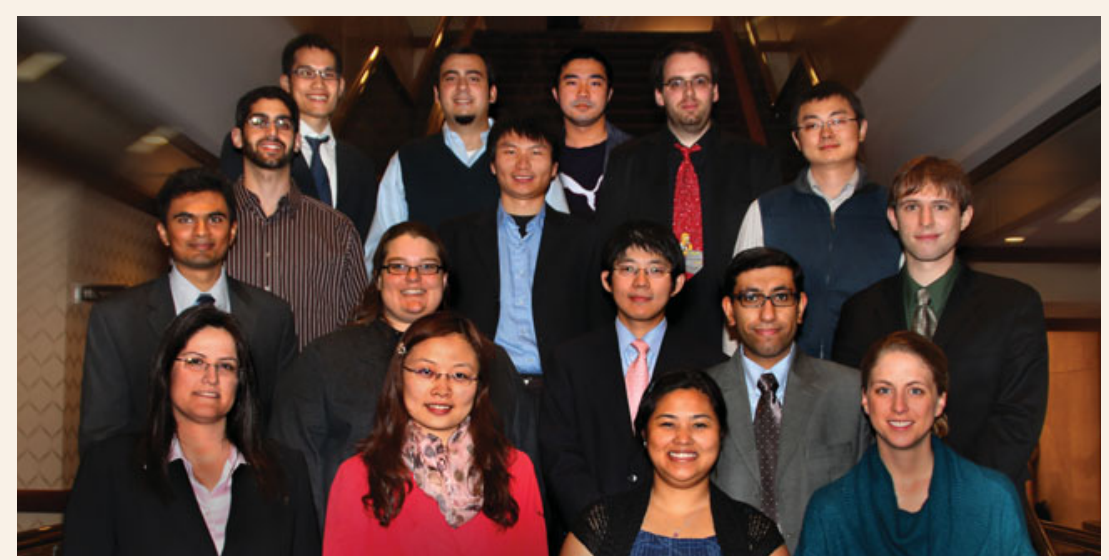

Silver Graduate Student Awards were awarded to (front row, left to right): Muge Acik (University of Texas at Dallas), Sa Zhou (Boston College), Rameshwori Loukrakpam (State University of New York at

Binghamton), and Laura Dickinson (Johns Hopkins University); (second row): Hassan Masoud (Georgia Institute of Technology); (third row, left to right): Pratap Rao (Stanford University), Amy Marconnet (Stanford University), Xiaoyan Li (Brown University), and Jonathan Singer (Massachusetts Institute of Technology); (fourth row, left to right): Kian Kerman (Harvard University), Jian Shi (College of Engineering, University of Wisconsin-Madison), and Chong Xie (Stanford University); (fifth row, left to right): Jin Suntivich (Massachusetts Institute of Technology), Mehmet Baykara (Yale University), Hailiang Wang (Stanford University), and Elad Mentovich (Tel Aviv University). Not shown: Akhilesh Gaharwar (Purdue University), Illan Kramer (University of Toronto), and Charles Sing (Massachusetts Institute of Technology). 
$\mathrm{In}_{2} \mathrm{O}_{3}$. Thin-film transistors from these oxide thin films now demonstrate high performance relative to competing materials used for flat-panel displays (such as a-Si), with channel mobility above $10 \mathrm{~cm}^{2} / \mathrm{Vs}$ and current on/off ratios in excess of $10^{9}$. Among these, transparent electronics were reported by $\mathrm{H}$. von Wenckstern (Universität Leipzig) utilizing $\mathrm{ZnO}$ channels and transparent electrodes including $\mathrm{Ag}_{x} \mathrm{O}$ Schottky gates or $p$-type $\mathrm{ZnCo}_{2} \mathrm{O}_{4}$ gates, yielding both excellent performance and enhanced stability over devices with gate insulators.

Anderson Janotti of UCSB compared the bandgaps of various oxide semiconductor materials like $\mathrm{ZnO}, \mathrm{SnO}_{2}$, and $\mathrm{TiO}_{2}$, which have bandgaps around 3.0 $\mathrm{eV}$. These materials are used in many applications such as transparent contacts, LEDs, laser diodes, transistors, photocatalysts, and gas sensors. The challenges that these oxide semiconductors pose include control of conductivity, bandgap engineering, heterostructures, epitaxial growth, and nanostructures. They are highly dispersive with wide bandgaps. The main goal of the group's research is to understand the role of doping in oxides using first principles.
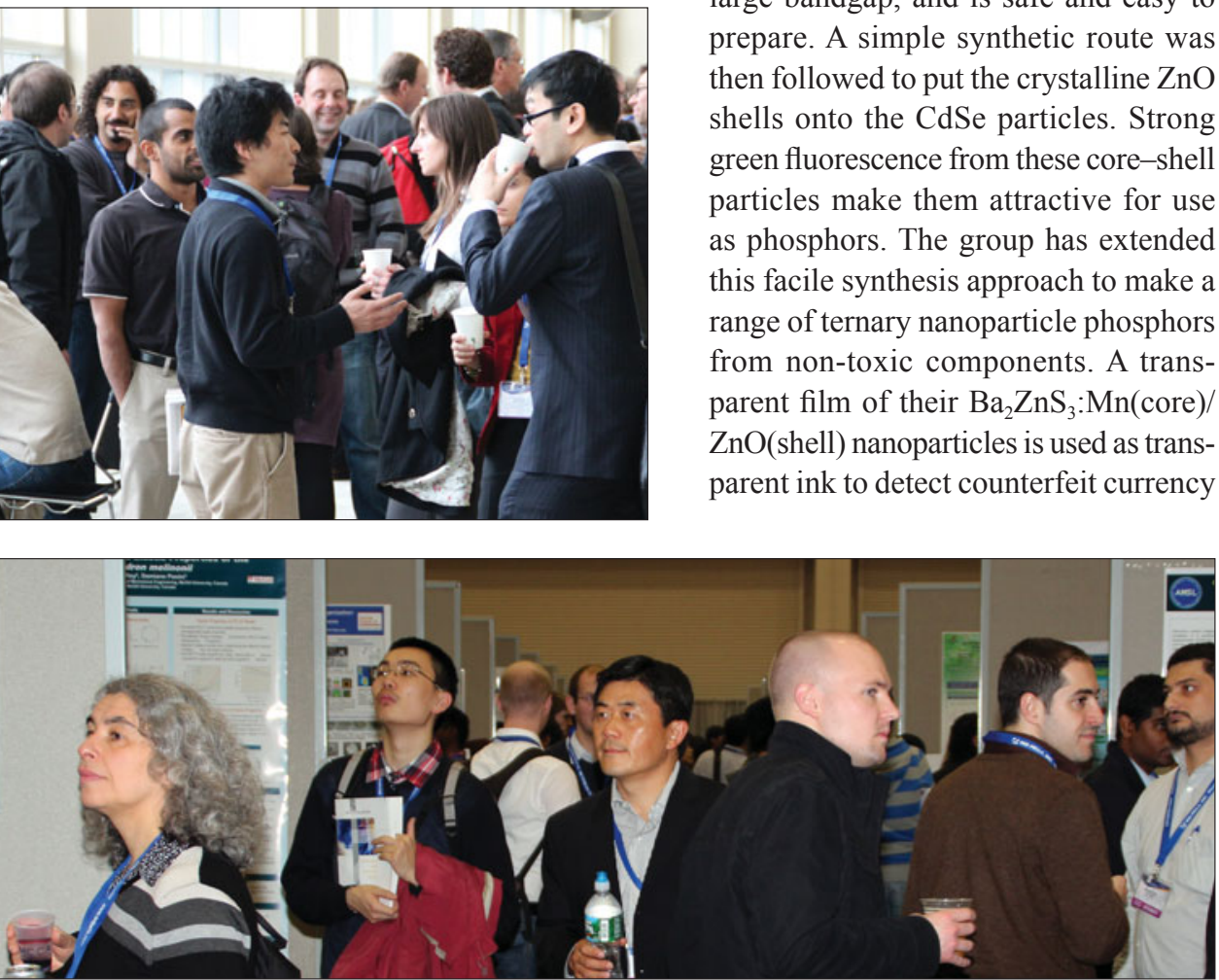

They experimented with different ways to study unintentional defects in these oxides. Hydrogen in $\mathrm{ZnO}$ can occupy interstitial and substitutional sites; in both forms, $\mathrm{H}$ is an acceptor and a donor, so it can cause $n$-type conductivity. Janotti said some progress has been observed in understanding unintentional $n$-type conductivity in $\mathrm{ZnO}$. Also nitrogen can act as a deep acceptor when substituted for some of the oxygen in $\mathrm{TiO}_{2}$.

In addition to doping effects, there has been great interest in the quantum confinement effect over the years. This interest has led to the synthesis of various nanoparticle phosphors such as $\mathrm{CdSe} / \mathrm{ZnS}$ core-shell nanoparticles. Masakazu Kobayashi of Waseda University, Japan, explored simple and scalable synthesis techniques to replace the complicated techniques and toxic raw materials generally used to make these nanoparticles. Their technique involved using a planetary ball mill to smash large CdSe starting materials into nanosized particles. Fluorescence measurements confirmed the quantum effect in these core CdSe nanoparticles. For the shell, Kobayashi's group sought to replace ZnS by $\mathrm{ZnO}$, as the latter has a reasonably large bandgap, and is safe and easy to prepare. A simple synthetic route was then followed to put the crystalline $\mathrm{ZnO}$ shells onto the CdSe particles. Strong green fluorescence from these core-shell particles make them attractive for use as phosphors. The group has extended this facile synthesis approach to make a range of ternary nanoparticle phosphors from non-toxic components. A transparent film of their $\mathrm{Ba}_{2} \mathrm{ZnS}_{3}: \mathrm{Mn}$ (core)/ $\mathrm{ZnO}$ (shell) nanoparticles is used as transparent ink to detect counterfeit currency and passports under UV light. Their red phosphors can improve the absorption properties of photodetectors, and will also be used for wavelength conversion in silicon solar cells.

But silicon has some competition as the material of choice in solar cells. Copper zinc tin sulfide (CZTS)/Se solar cells are candidates to reduce overall PV module cost, as more than $40 \%$ of the cost of silicon solar cell modules comes from the silicon wafer. While CdTe-based systems have been successful recently, there is considerable interest to replace toxic elements like cadmium with elements like copper. This was the driving force behind research on CIGS (copper indium gallium diselenide) based solar cells. The scarcity and cost of indium are now encouraging research on a new class of solar cells based on CZTS. Being a direct bandgap material, CZTS has a strong absorption coefficient, and the bandgaps can be tuned by replacing sulfur with selenium. In Symposium $\mathrm{O}$ on compound semiconductor-related work in energy, Louis Grenet of CEA, Grenoble, explored the creation of $6 \%$ efficient CZTS/Se solar cells by combining ZnS sputtering, followed by coevaporation of copper and tin. The resulting films are then annealed in a selenium atmosphere to obtain the CZTS/Se films.

\section{Nanomaterials}

In Symposium AA on carbon nanotubes, graphene, and related nanostructures, Motoo Yumura of AIST-Nanotube Research Center in Japan spoke on recent mass production of single-walled carbon nanotubes (SWCNTs) and expectations in the near future both in research and industry. Yumura's institution concentrates on the development of techniques for controlling, separating, and characterizing SWCNTs. The idea is to use metal semiconductors to separate SWCNTs. Also, new techniques such as a gel column have the potential for high throughput.

The Center also focuses on technology development for dispersion of SWCNTs. This involves the thermalconductive supergrowth (SG) of SWCNT rubber. These composite resin/ 
rubbers have high thermal conductivity and are good dispersants. A third major area of the Center is safety management of nanomaterials. The goal is to develop a quick and simple method for toxicity assessment, such as on-site measurement.

Yumura said that the current mass production technique for producing SWCNTs is by direct injection pyrolytic synthesis (DIPS). Though simple, it does not produce high-quality industrial materials, and thus requires post-treatment such as purification or modification. But by precisely controlling the reaction conditions in the DIPS method, synthesis was successfully achieved by Yumura and colleagues. The result is high-strength fiber SWCNTs with a diameter control with $0.1 \mathrm{~nm}$ precision. Kenji Hata (AIST) discovered the SG technology based on a novel growth technique whose basic elements are ethylene and water. It allows ultrahigh efficiency synthesis at a rate 1000 times faster than current technologies, and ultrahigh purity of $99.88 \%$.

In a presentation given in Symposium DD on organic nanocomposites for thermoelectric energy conversion, Choongho Yu of Texas A\&M University considers carbon-based organic composites to be excellent for thermoelectric energy harvesting. Current thermoelectric devices with semiconductors connected in series are used for both cooling and energy harvesting. These state-of-theart thermoelectric devices contain a $\mathrm{Bi}-\mathrm{Te}-\mathrm{Pb}-\mathrm{Sb}$ alloy, which exhibits good properties but is very inexpensive and toxic. So Yu and colleagues decided to try harvesting energy using polymers, which are inexpensive, light, flexible, and non-toxic. However, polymers tend to be poor electrical conductors, while thermoelectric conversion requires high electrical conductivity and low thermal conductivity. The researchers achieved these parameters using SWCNTs and decoupling conductivities. They controlled the junctions and surfaces by coating the SWCNTs, which caused the transfer property to decrease dramatically. Also by introducing PEDOT connections between SWCNTs it is possible to tailor the device for a specific application, Yu said.

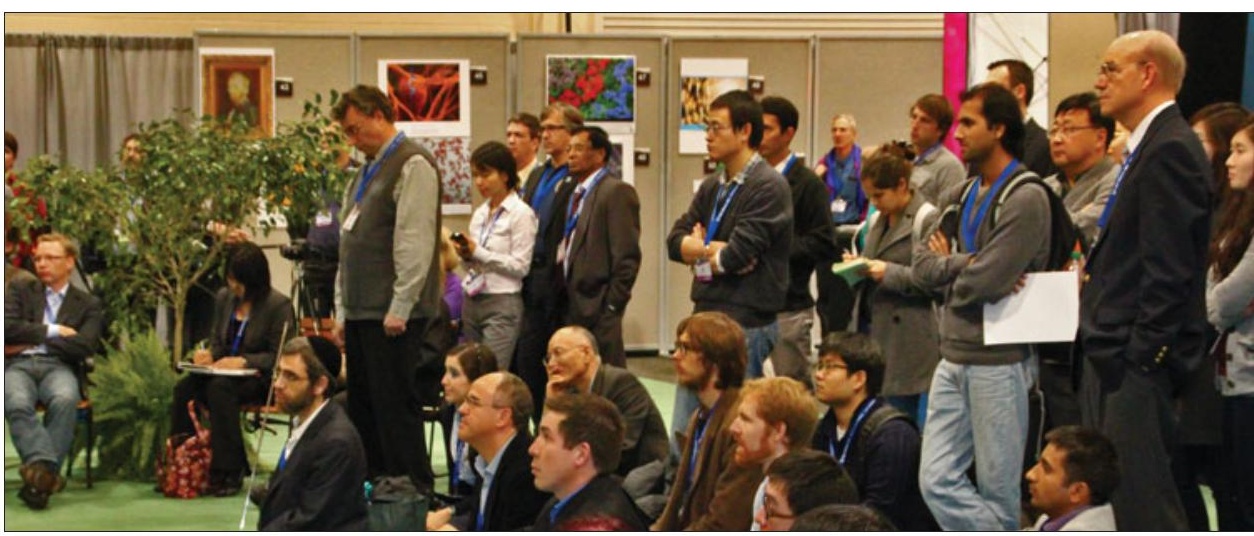

\section{Awards and special events}

Complementing the research reported in the symposia were additional technical talks presented by MRS award recipients as well as speakers in Symposium $\mathrm{X}$ consisting of broad overviews for the technical nonspecialist. The Meeting also hosted sessions on professional development and on government funding opportunities.

A. Paul Alivisatos of Lawrence Berkeley National Laboratory received the MRS Von Hippel Award, the Society's highest honor, in recognition of his work on colloidal nanoparticles. "[The Greek philosopher] Democritus conceived of the atom through successive division of matter, thus setting the stage for the (eventual) study of scaling laws for nanomaterials," Alivisatos said at the beginning of his talk. Alivisatos has found scaling laws to be intriguing and inspirational throughout his scientific career; they have led him to investigate numerous aspects of nanoparticles over the years, culminating in the synthesis of artificial atoms and molecules.

His eventual success at synthesizing high-quality colloidal CdSe quantum dots stemmed from a scaling law: the melting temperature decreases as particles get smaller, largely because there are very few bonds to break at the nanoscale. "The low melting temperature is what enabled synthesis of quality nanoparticles in a hot organic solution," Alivisatos said. "In such small particles, impurities can anneal themselves out."

Ultimately he and his colleagues began thinking of colloidal quantum dots as artificial atoms, and they won-

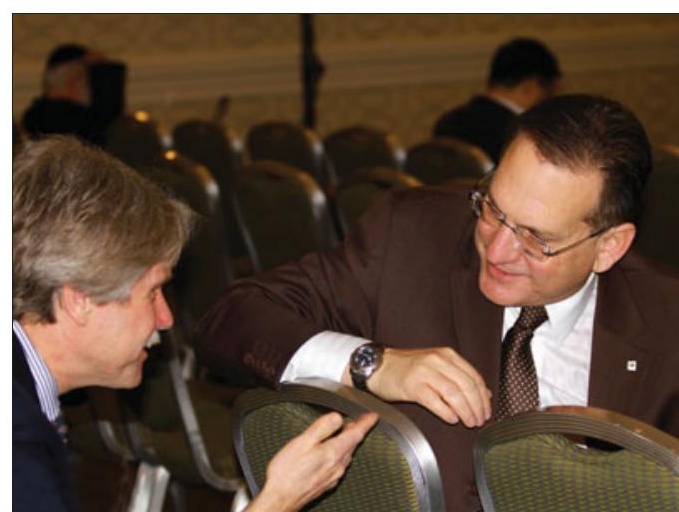

dered whether they could build artificial molecules. "In the early days, when we wanted to bring quantum dots together, we didn't have the means," Alivisatos said. "So we turned to biology."

Soon they were using DNA to couple three artificial atoms into what he called a "mickey-mouse-shaped" artificial molecule. Recently, the Alivisatos group made a tetrahedron of DNA with different-sized nanocrystals at each corner, effectively creating an artificial, chiral nanocrystalline molecule.

The DNA-based work was fascinating and useful, but Alivisatos had wanted to produce artificial molecules inorganically all along. After some experimentation, he and his colleagues learned how to take existing inorganic nanoparticles and perform chemical operations on them to promote extension and branching. With these chemical methods, they began to control the symmetry, topology, connectivity, and directionality of these artificial inorganic molecules. Because of the photoluminescent properties of these quantum dot nanoparticles, they could see a redshift of the light from one arm of a tetrapod struc- 
ture when it experienced tensile stress, making it an excellent strain sensor.

Alivisatos concluded by revealing that they have been watching nanoparticles grow inside electron microscopes, using a liquid cell with graphene windows to spy on the nucleation and growth of the nanocrystals. As amazing as this seems, he was, like Democritus, philosophical about these developments.

"Hopefully everything I showed you here tonight will be obsolete in 10 years because we will have developed better techniques," Alivisatos said.

While graphene has been touted for its exceptional electronic and optical properties, this year's David Turnbull Lecturer Phaedon Avouris of the IBM T.J. Watson Research Center warned that most of the research on graphene isolates the material from its environment. While he does not dispute the extremely high carrier mobility, the excellent heat conduction, and the wide range of optical absorbance of graphene, Avouris carefully pointed out that graphene "is all surface. In contact with other materials, its properties are perturbed."

Avouris is very positive on the use of graphene in optoelectronics because it absorbs at least $22 \%$ of any wavelength in the spectrum. Also, its Fermi level

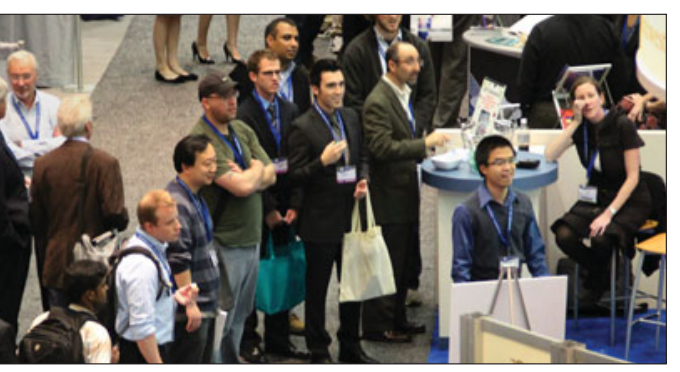

can be tuned using an electric field, and shows high-speed photoresponse. His research has shown no decrease in this photoresponse up to $40 \mathrm{GHz}$, the limit of his measurement system, in a singlecontact graphene photodetector. Avouris said graphene will have its greatest impact in optoelectronics in the terahertz frequencies.

The critical issues for graphene, according to Avouris, are producing high-quality, large-area, low-price materials; controlling doping; developing low-resistance contacts and gates; and producing ultrathin, high-dielectricconstant insulators that are compatible with graphene. He concluded his talk by urging researchers to "work in real world conditions with graphene, not just at microkelvins."

This year's MRS Medalists, Peidong Yang of the University of CaliforniaBerkeley and Z.L. Wang of the Georgia Institute of Technology, are both using their areas of research to help solve the global energy issue. Yang, recognized for his work on nanowire research, began to conceive a "terawatt solution" that involved waste heat recovery and storage of energy from renewable sources. A typical thermoelectric cogeneration power plant loses $60 \%$ of its energy as waste heat, according to Yang. His research led to solution-processed rough Si nanowires with a thermoelectric figure of merit $Z T$ that is 100 times higher than typical Si nanowires. This makes the solution-processed nanowires good candidates for thermoelectric power generation, converting waste heat into electricity. Yang founded a company called Alphabet Energy in 2009 in order to move this technology to commercialization.

For energy conversion, Yang is relying on earth-abundant elements synthesized into nanowires by "mild chemical" methods. The device he is trying to build is a nanowire-based dye-sensitized solar cell, with single-crystal nanowires used as the electrodes. He produces $\mathrm{CdS}$ $\mathrm{Cu}_{2} \mathrm{~S}$ core-shell nanowires by dipping a $\mathrm{CdS}$ nanowire in a $\mathrm{CuCl}$ solution for $10 \mathrm{~s}$ at $50^{\circ} \mathrm{C}$ (mild chemistry). The coreshell nanowire has a coherent interface, which leads to a high fill factor and a high open-circuit voltage. This coherent interface is a key structural requirement for a high-junction area solar cell, Yang said. His next step in this effort is to find an element to replace $\mathrm{Cd}$ to achieve his earth-abundant, nontoxic product goals.

Z.L. Wang discussed his most recent work on piezophototronics, which results from the three-way coupling between piezoelectricity, photonic excitation, and semiconductor transport, allowing for the tuning and control of electro-optical processes by strain-induced piezopotential. The "piezophototronic effect" has been applied for raising the sensitivity of UV sensors by 300-500 times, enhancing light-emitting diode external efficiency by $325 \%$, and raising high solarcell efficiency by $50 \%$. The development of piezophototronics promises to have great impact on energy science and optoelectronic devices fabricated using $\mathrm{ZnO}$ and $\mathrm{GaN}$, said Wang.

In a Symposium X presentation that investigated both extremes - the highs and the lows - of thermal conductivity
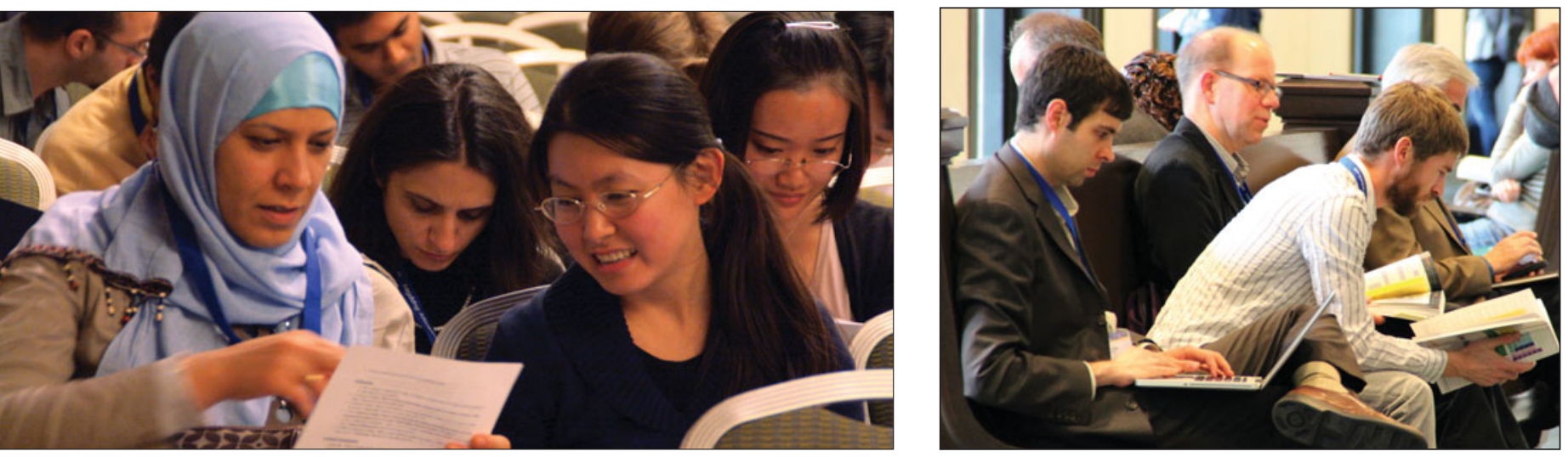
in materials, David Cahill of the University of Illinois at Urbana-Champaign described how his team's work using timedomain thermoreflectance (TDTR) has enabled them to study materials across the conductivity spectrum. The technique uses thermal waves to measure transport properties, but because they are usually examining nanoscale materials the measurement must be ultrafast. Fully understanding thermal conductivity is difficult because the solution "involves an integral over all thermally excited states in a material," Cahill said.

Using TDTR measurements, Cahill's group showed that a $60-\mathrm{nm}$ film of $\mathrm{WSe}_{2}$ has the lowest thermal conductivity observed in a fully dense solid, having only twice the thermal conductivity of air. This is a factor of six less than the calculated amorphous limit for this material. Being an optical technique, TDTR also allows them to study thermal conductivity of materials under extreme conditions, such as ice VII (a cubic crystalline phase of water ice formed at high pressures) at $20 \mathrm{GPa}$. When compressed to two-thirds of its normal volume, ice VII had a high thermal conductivity, close to that of sapphire.

Cahill's group is now investigating what he calls "extraordinary spin-wave thermal conductivity in $\mathrm{Ca}_{9} \mathrm{La}_{5} \mathrm{Cu}_{24} \mathrm{O}_{41}$." The copper oxide spin ladders are believed to carry the heat, but this is still a work in progress. His group plans to use TDTR's frequency dependence to probe magnon-phonon coupling in this material. "Time-domain thermoreflectance enables experiments that were not possible a decade ago," Cahill said.

In another Symposium X talk, Hideomi Koinuma of the University of Tokyo began by saying, "Today I am going to tell a story," and took the audience in Symposium X on a "universal dream" in which large areas of solar panels sprawled out in deserts all over the world generate energy and power for the rest of the planet using high- $\mathrm{T}_{\mathrm{c}}$ superconducting cables. Proposed at the G8+5 Academies' meeting in Rome in 2009 by the Science Council of Japan, the Sahara Desert solar breeder (SSB) plan is the first step in realizing such a global clean energy superhighway. A similar project called

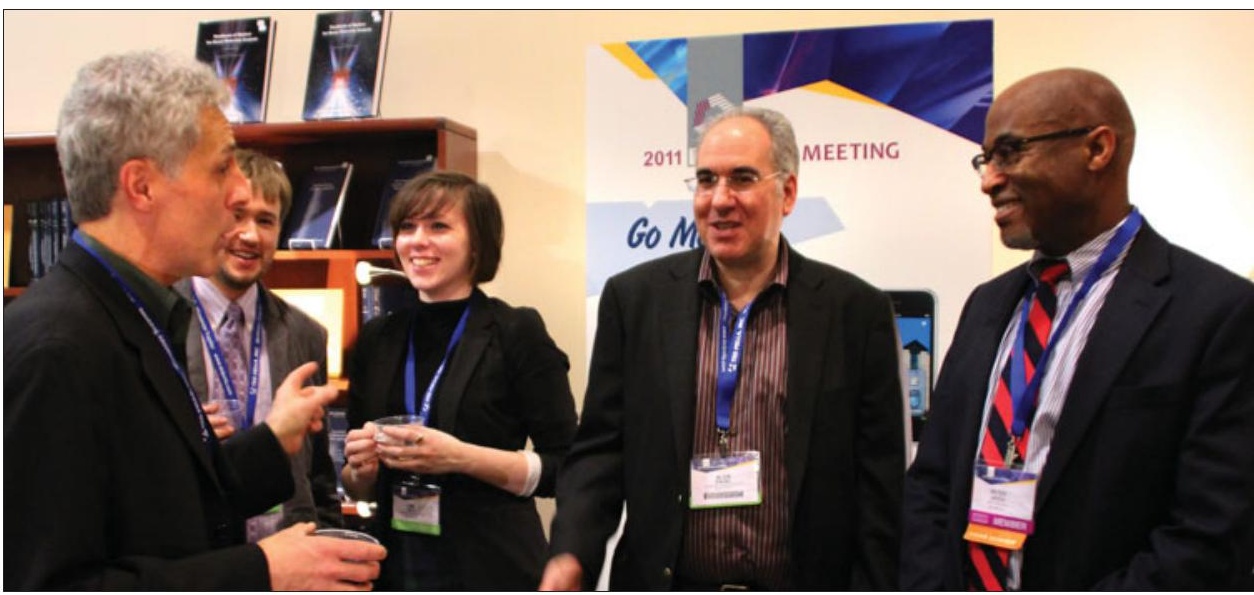

Desertec is already moving forward to generate solar power in the deserts of North Africa and the Middle East. Using this abundant "natural energy source," however, is easier said than done, mainly owing to cost and policy-related issues, said Koinuma.

"Based on my experience over 30 years, I am now coming back to crystalline silicon solar cells," Koinuma said, while large portions of the PV community shift farther away from silicon PV research. But his reasoning is that only silicon solar can easily provide $>100$ $\mathrm{GW} /$ year of clean, renewable power. The SSB project proposes to erect around 100 solar breeder reactors that can provide over $30 \%$ of the energy demand through high-temperature superconducting cables. Another ambitious idea is a solar pyramid system, based on $>14 \%$ efficient silicon PV modules.

In a rare Symposium $\mathrm{X}$ panel presentation, four officials from the White House and funding agencies gave an overview of the Materials Genome Initiative (MGI) that was announced by the White House last summer. Cyrus Wadia, assistant director of Clean Energy and Materials R\&D in the White House Office of Science and Technology Policy, started the conversation by saying that the initiative's motto is "two times faster and two times cheaper." That is, the government wants to enable researchers to develop and bring new materials to market in half the time and at half the cost compared to past efforts, which typically took 20-30 years from laboratory to market. To do this, the

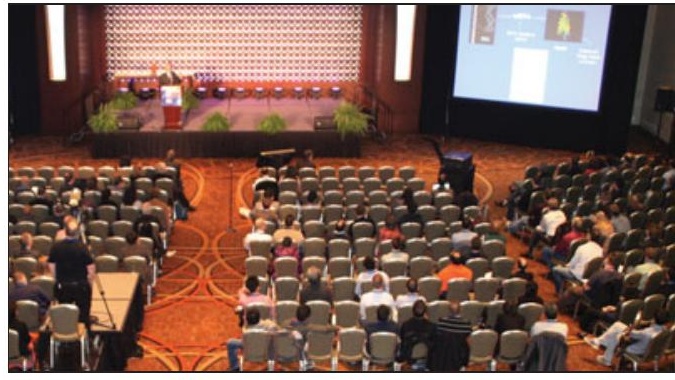

government is establishing a "Materials Innovation Infrastructure" that includes the areas of computational tools, experimental tools, and digital data. The plan is to bring in the software community to create open platforms and universal access to computational tools to enable materials researchers to replace some experiments with simulations. Also, experimentalists need to develop new techniques and new figures of merit to bridge Regarding digital data, Wadia said, "We believe that data transparency encourages innovation." There is currently no repository for large amounts of materials data, so a new "ecosystem" will be built to encourage data sharing.

Panelist Harriet Kung, director of Basic Energy Sciences in the Department of Energy (DOE), said that the recommendation given to the White House based on workshops held by DOE was that the federal government needs to maintain a long-term stewardship of integrated, sustainable software as an investment in the materials science community. Ian Robertson, director of the Division of Materials Research at the National Science Foundation (NSF), followed with a the experimental/computational divide. 
